\title{
Design and Development of Semi-Automatic Stretcher Robot
}

\author{
G. Shanmugasundar, E. Aswin, R. Danesh, G. Prabagaran, M. Yohapraveen \\ Associate Profes sor Mechanical Engineering, Sri Sai Ram Institute of Technology, Chennai, Tamilnadu, India. \\ Department of Mechanical Engineering, Sri Sai Ram Institute of Technology, Chennai, Tamilnadu, India. \\ ${ }^{*}$ Corres ponding author Email: shanmugasundar.mech@sairamit.edu.in
}

\begin{abstract}
The wheelchair and stretcher are generally used in hospitals, airports, train stations, shopping malls, etc. This design here is a modified wheelchair with stretcher as needed. This machine can be used to convert the wheelchair to a stretcher as needed. It is accessible semi-automatically. The chair transforms into a stretcher when the rocker switches are pressed by using electric actuators. The stretchers can be detached from the main frame for the convenience of the patient and clinician, facilitating patient access with less effort and transport. The folding mechanism makes it easy to store a large number of stretchers inserted as chairs in a relatively smaller space. The number of patients around the world is increasing day by day. Thus, in hospitals, patients must be moved from wheelchair to stretcher, from stretcher to bed, from bed to wheelchair or vice versa; which creates dangerous conditions for patients. A wheelchair with stretcher is required to facilitate the mobility of the disabled patient and to provide new medical equipment for use in hospitals.
\end{abstract}

keyword: semi-automatic, easy operating, foldable, convertible, cost effective.

\section{Introduction}

A wheelchair is a chair with wheels. The device is available in versions permitting both manual propulsion via way of means of the seated occupant turning the rear wheels via way of means of hand, or electric powered propulsion via way of means of automobiles and actuators for leg rest and back rest. There are regularly handles in the back of the seat to permit it to be driven via way of means of every other person. Wheelchairs are utilized by human beings for whom on foot is hard or not possible because of illness, injury, or disability. People who've is sue sitting and on foot regularly employ a wheel bench. Chair and wheel have been the earliest innovations of man. A wheelchair is a wheeled mobility tool designed specifically for disabled individuals. The tool is propelled both manually or thru diverse semi-computerized systems. Wheelchairs are utilized by human beings for whom on foot is hard or not possible because of illness (physiological or physical), injury, or disability. Early wheelchairs have been supposed handiest to assist a disabled character to transport from one location to every other however nowadays the wheelchairs are taken into consideration as now no longer handiest for the transportation cause however additionally a manner to explicit users' individuality. In India the wide variety of disabled populace had a super increase with inside the beyond few years. Huge quantity of human beings has congenital disabilities, all other few chances are the sufferer of injuries and diverse sort of mobility gadgets are inevitable a part of their life.

\section{Review of Lite rature}

People have disabilities with their hands, foot and lower extremities because of which they are unable to perform regular tasks. Many technologies are available to overcome this problem. To overcome this problem, there are several applications in the market which help handicapped people to perform their tasks. Proposed design supports voice activation system for physically disabled persons incorporating manual operation. If a person is handicapped, they are dependent upon others for their day-to-day operations such as orientation etc. Several studies have shown that the independent mobility, which includes power wheelchair, manual wheelchair and walker access the benefit to both children and adults. Independent mobility reduces dependence on caregivers and family members and promotes feelings of self-reliance. Impaired mobility often results in decreased opportunities to socialize, which leads to social isolation, an xiety and depression. While the needs of many individuals with disabilities can be satisfied with traditional manual or power wheelchair, a segment of the disabled community finds it difficult or impossible to use wheelchairs independently. This population includes individuals with low vision, cognitive deficits, etc. The proposed voice-controlled wheelchair would bring more convenience for the disabled people. The technology can also enhance safety for users who use ordinary joystick-controlled powered wheelchairs, by preventing collisions with walls, fixed objects, furniture and other people. There are many advantages to such a system like It reduces human efforts. This is helpful to physically handicapped people who could not able to operate home appliances with their hand. This will help to save energy to some extent, since some people feels lazy to go and switch off the appliances manually. It is easy to operate for the people who are tried and does not need to operate the home appliance manually by hands. It reduces risk. The proposed system has certain limitations too such as: It requires extra supply to operate the model. 


\section{Proposed System}

Modern day wheel chairs comprise light materials, microprocessor controlled and lots of greater sophisticated systems. There is a revolution of wheelchairs to be had today pushed with the aid of using needs and preference or guy today. The future expects a higher variety of wheelchairs that might in shape the creativeness of the human thoughts and serve the needy. The primary shape of the wheelchair includes various parts. In simple words it's not anything however a set of wheels connected to a chair. There are some vital matters a wheelchair should comprise. A seat should be co mfortable, in order that the character does now no longer get worn-out sitting on it for a long time. It should comprise a backrest that offers a good lu mbar support. It should have an arm rest at an optimum height and an additionally a foot rest. The maximu $\mathrm{m}$ ital assume is it should have brakes for the wheels. Since the birth of the wheelchair there were many changes in its design. Today there exists a large kind of wheelchairs - manually, electric, or self-propelled, foldable or rigid. Apart from those they are labeled primarily based totally on their usage, status wheelchair, sports wheelchair, mobility scooters, bathroom wheelchair, steps hiking wheelchair etc.

\section{Hardware Description}

The major component involved in the construction of the wheelchair cum stretcher are: Chassis Rear Wheel Casters Bolt and Nut Main base structure Bearing Rack and Pinion Gear. chassis The base is essentially a platform or it could additionally be known as a guide for the complete assembly. The base is built with the assist of channels manufactured from metals which includes aluminum, stainless metal, moderate metal etc. In the layout of this wheelchair, the bottom includes a shape known as a body manufactured from moderate metal. A sheet of metallic is constant at the top surface of the body which acts as a platform for the individual to sit. The sheet is essentially welded to the body with the assist of welding method. The form of welding employed on this layout is arc welding. When the sheet metallic is mixed with body it forms a rig id shape so known as base. rear wheel The rear wheel which is connected to each other using the shaft, so as to keep it in align ment. the wheel is of diameter $69 \mathrm{~mm}$. this wheel will be hold ing the weight of whole body including the human weight. casters The caster wheels are attached to the body of the chair with the help of end bearing and bearing caps. The wheel is made up of fiber wheel inch diameter. A caster (or castor) is an driven, single, double, or compound wheel that is designed to be mounted to the bottom of a larger object (the "vehicle") so as to enable that object to be easily moved. They are available in various sizes, and are commonly made of rubber, plastic, nylon, alu minu m, or stainless steel. Here we are providing with two caster wheels in the front for proper balancing and also for changing direction as convenient. main base structure This is the main base with the seat platform on it. Here the base is welded to the shaft of the wheels. The wheels are attached using the nut and bolts, so its removable. The casters are also bolted to the base leg, so as to keep it removable in case we need to change the caster. bearings The bearings are pressed smoothly to fit into the shaft because if hammered the bearing may develop cracks. Bearing is made of steel material and bearing cap is mild steel. Ball and roller bearings are used widely in instruments and machines in order to minimize the friction and power loss. While the concept of the ball bearing dates back at least to Leonardo da Vinci, their design and manufacture has become remarkably sophisticated. This technology was bought to its present state of perfection only after a long period of research and development. The benefits of such specialized research can be obtained when it is possible to use a standardized bearing of the proper size type. rack and pin ion arrangement The block is the important part of the unit as it houses the rack and pinion. This block converts the linear motion into rotary motion. Rack and pinion gear system is used to transmit rotary motion into linear motion. The rack is a portion of a gear having an infinite pitch diameter and the line of action is tangent to the pinion.

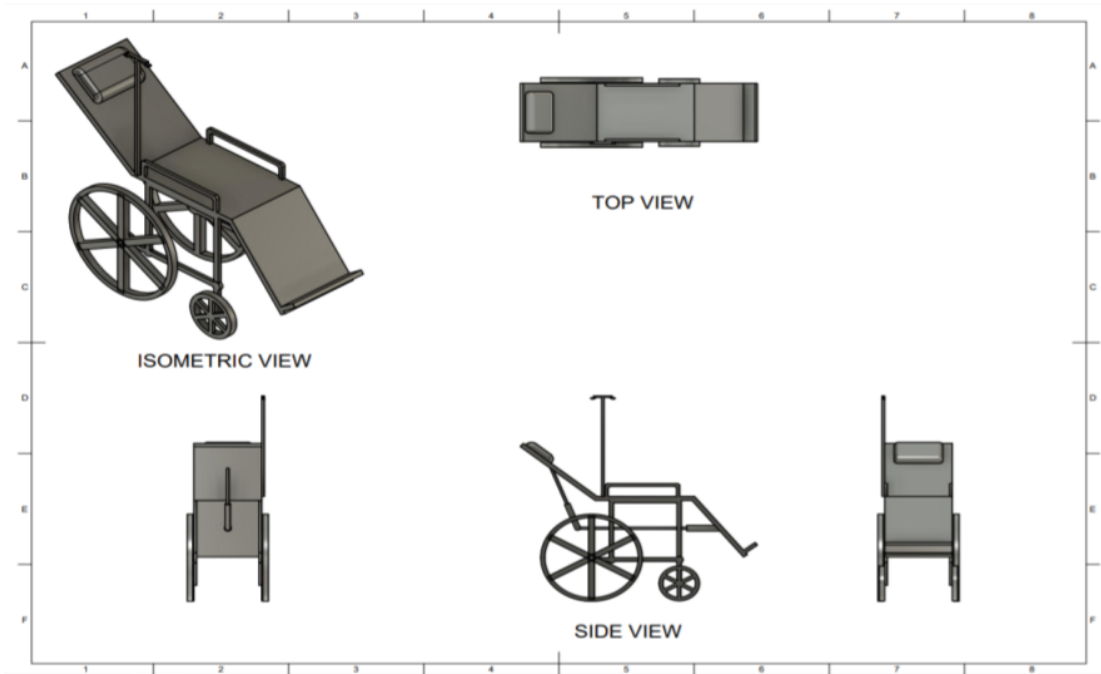

FIGURE 1. CAD model of the proposed robot 


\section{Analysing The Problem}

This is a basic model of wheelchair that are commonly used. These can only be used to move the patients in a seating posture with limited comfort. It's difficult to move the Patients to a separate stretcher. This design basically consists of limited mobility. Here it is difficult for the patients by themselves to move on to a bed in need of some sleep and comfort. The main problem we faced is, which type of material we should use, after few failures we conclude that low weight and high strength material we should use. Strength of frame of the wheelchair base to hold heavy body. Strength of structure of the stretcher. How many linkages. Placing of the mechanis $m$. Proper spacing for alteration in design during development. Placing of wheels. Locking systemfor the stretcher to wheelchair. Joints and hinges

\section{Results and Dis cussion}

The conventional wheelchair cum stretcher has numerous drawbacks and its expensive too. The available wheelchair doesn't provide the provision for stretcher. So, to overcome all these drawbacks, this new model of wheelchair cum stretcher is designed. The advantages of this design compared to conventional wheelchair are listed below. Maintenance is easy and hence make it more effective. Detachable stretcher is provided, giving comfort in transferring the patient from one bed to another. Even after detaching, the base can be used as a chair. Construction of this design is simple. The extra wheels on the stretcher will be helpful to use it as trolley. This model is cheaper than the model in the market. Scope of the project this system will be a real-time voice controlled wheelchair for the physically disabled person. This system will be designed to operate the wheelchair based on the voice of the user and control the movement according to the command given by the operating person. The voice would be given through a unilateral mic and would be converted into binary format by voice recognition kit. Thus, this binary format would be checked with the binary code fed to the microcontroller, if true the command will be performed. More specifically, this system is designed to allow an admin and users to give the voice command to the wheelchair. This command would be performed within seconds. On the whole it's basic operation would be left, right, stop, go, back. Basically, it's a wheelchair controlled by voice. Advantages the patients like quadriplegic and cerebral palsy, lack of force, can easily handle this voice-controlled system. The use of Arduino makes the programming of the system easy and thus, reduced the software and hardware interfacing problems. The system can be operated by giving synthetic voice commands. The system is fully automated because of the use of Arduino and motor drivers.

\section{Conclusion}

The mechanism is designed and developed in order to reduce the human fatigue. Wheelchairs are now considered not only means of transportation but also as a way to allow users to express their individuality. Also allowing the helper of nurse to ease in handling the patient in severe cases. When the patient is required to transfer from bed to wheel chair from one place to hospital or any other place it becomes very difficult by nursing staff as well as patient also. Due to the transferring from bed to wheel chair or vice versa, stresses are developed in the body of patient and as well as nursing. The above problems which are generated at the timing of patient transferring from bed to wheel chair can be eliminated by developing new design of stretcher cum wheelchair i.e. providing the wheelchair cum stretcher with a detachable stretcher which can operated easily as well as used as a trolley when needed. The cost of this design may be little higher than the common wheelchair, but this design has many more features that can be helpful for the patient as well as the nursing staffs. Also, we have understood that there are many scopes for future improvements.

\section{References}

1. Richard C. Simpson Phd, "Smart Wheelchairs”,Department Of Rehabilitation Science And Technology, University Of Pitts burgh,Pa(2005)

2. Sumedh. J. Suryawanshi, Dr. K. Janardhan Reddy "Product Development Of Wheelchair For People Disabled In Legs", (2013) (Smbs2013)

3. Roger Bostelman, James Albus“A Multipurpose Robotic Wheelchair And Rehabilitation Device For The Home”, (NistGaithersburg), (2007)

4. Prof.R.S.Nipanikar, Vinay Gaikwad, Chetan Choudhari, Ram Gosavi, Vishal Harne"Automatic Wheelchair For Physically Disabled Persons", (2013) (Ijarece)

5. Sreerag, Gopinath, Manas Ranjan Mishra "Design And Development Of Conceptual wheelchair Cum Stretcher", School Of Advanced Studies, Bangalore, (2011)

6. Shanmugasundar, G., Dharanidharan, M., Vishwa, D., \& Sanjeev Kumar, A. P. (2021). Design, analysis and topology optimization of connecting rod. Materials Today: Proceedings, 46, 3430-3438.

7. G Shanmugasundar, M Dharanidharan, D Vishwa, A Jayaprakash and P Abimanyu , 2020, "Design and Finite Element Analysis of Prosthetic Hand Controlled by Wireless Gestures for Differently-abled People" IOP Conference Series: Materials Science and Engineering 923 (2020) 012019 IOP Publishing, Doi:10.1088/1757899X/923/1/012019.

8. G. Shanmugasundar, G. Fenneth Moses, S. Jayachandran, V.D. Rathnavel Subramanian and R. Rajagopalan, 2020 
"Design and Fabrication of Solar Powered Multi-Purpose Agricultural Vehicle with IOT Control" , Journal of Advanced Research in Dynamical and Control Systems, Volume 12, Pages: 1928-1933.

9. G.Shan mugasundar, R.Yokesh, S.Yuvaranjith, R.Barath, S.Balasubramanian (2020), Design and Fabrication of Intelligent Gas stove for women safety , International journal of pharmaceutical research , https://doi.org/10.31838/ijpr/2020.12.02.0126

10. G.Shanmugasundar, M.Dharanidharan,, D.Vishwa, P. Gokul, (2020), Design, Modelling and Analysis of an Autonomous Ornithopter Robot for Env ironmental Pollution Mon itoring, Interciencia Journal (ISSN: 0378-1844), 45(12 ), PP- 2-10. https://intercienciajournal.com/intercien/index.php/pdf/stream/54un4/1604942593.

11. G. Shan mugasundar, P. Jagadeeshwar, S. Adithya, V. Nagappan, and M. Bhaskar, "Design, fabrication and analysis of personal vacuum assisted climber," in Journal of Physics: Conference Series, 2019, vol. 1362, no. 1, doi: 10.1088/1742-6596/1362/1/012057.

12. G. Shan mugasundar, R. Sivaramakrishnan, S. Meganathan, and S. Balasubramani, "Structural optimization of an five degrees of freedom (T-3R-T) robot manipultor using finite element analysis," in Materials Today: Proceedings, 2019, vol. 16, pp. 1325-1332, doi: 10.1016/j.matpr.2019.05.231.

13. G. Shan mugasundar, R. Sivaramakrishnan, and S. Balasubramani, "Method of Trajectory Generation of a Generic Robot using Bresenham's Circle Algorithm," Indian J. Sci. Technol., vol. 9, no. 48, 2017, doi: 10.17485/ijst/2016/v9i48/108476.

14. R. Sridhar, G. Shanmugasundar, and A. Srithar, "A Geometrical Modular Design for Handling of LPG Cylinders using Nested Kinematic Robotic Gripper," in Indian Journal of Science and Technology, 2016, vol. 9, no. 48, doi: 10.17485/ijst/2016/v9i48/108474.

15. Kalita, Kanak, Uvaraja Ragavendran, Manickam Ramachandran, and Akash Kumar Bhoi. "Weighted sum multiobjective optimization of skew composite laminates." Structural Engineering and Mechanics 69, no. 1 (2019): 21 31.

16. G. Shan mugasundar and R. Sivaramakrishnan, "Design and analysis of a newly developed seven degree of freedom robot for inspection," Int. J. Control Theory Appl., vol. 9, no. 24, pp. 393-402, 2016.

17. G. Shanmugasundar and R. Sivaramakrishnan, "Computer aided modelling and simulation of a generic ro bot for inspection,”Int. J. Appl. Eng. Res., vol. 10, no. 24, pp. 44049-44056, 2015.

18. G. Shan mugasundar, R. Sivaramakrishnan, R. Sridhar, and M. Rajmohan, "Computer Aided Modelling and Static Analysis of an Inspection Robot," in Applied Mechanics and Materials, 2015, vol. 766-767, doi: 10.4028/www.scientific.net/amm.766-767.1055.

19. G. Shan mugasundar, R. Sivaramakrishnan, and M. Rajmohan, "Computer aided simulation for workspace plot of a newly designed inspection robot," in 2014 IEEE International Conference on Computational Intelligence and Computing Research, IEEE ICCIC 2014, 2015, doi: 10.1109/ICCIC.2014.7238470. 